versely if the first row is multiplied by the inverse of $c\left(\bmod p^{k}\right)$. This inverse exists, and the correspondence is one-to-one, because $c$ is prime to $p$. This proves (3).

The sum of the probabilities $P_{n}\left(a p^{\alpha}, p^{k}\right)$, where $a$ runs through the values $1,2, \cdots, p^{k-\alpha}$, is clearly the probability that a determinant be divisible by $p^{\alpha}$. The terms of this sum can be simplified and collected by use of (3), and we have

$$
P_{n}\left(0, p^{\alpha}\right)=\sum_{r=0}^{k-\alpha} \phi\left(p^{k-\alpha-r}\right) P_{n}\left(p^{\alpha+r}, p^{k}\right)
$$

Replacing $\alpha$ by $\alpha+1$, and subtracting the resulting equation from (11), we arrive at (4).

Purdue University

\title{
ON THE NOTION OF THE RING OF QUOTIENTS OF A PRIME IDEAL
}

\section{CLAUDE CHEVALLEY}

Let $\mathrm{D}$ be a domain of integrity (that is, a ring with unit element and with no zero divisor not equal to 0 ), and let $\mathfrak{u}$ be a prime ideal in $\mathfrak{D}$. We can construct two auxiliary rings associated with $\mathfrak{u}$ : the factor ring $\mathfrak{D} / \mathfrak{u}$, composed of the residue classes of elements of $\mathfrak{o}$ modulo $\mathfrak{u}$, and the ring of quotients $\mathfrak{D}_{\mathfrak{u}}$, composed of the fractions whose numerator and denominator belong to $\mathfrak{D}$, but whose denominators do not belong to $\mathfrak{u}$. These constructions are of paramount importance in algebraic geometry; if $\mathfrak{D}$ is the ring of a variety $V$, there corresponds to $\mathfrak{u}$ a subvariety $U$ of $V ; \mathfrak{o} / \mathfrak{u}$ is the ring of $U$, whereas the ring $\mathfrak{o}_{\mathfrak{u}}$ is the proper algebraic tool to investigate the neighborhood of $U$ with respect to $V$.

Now, the local theory of algebraic varieties involves the consideration of rings which are not domains of integrity (this, because the completion of a local ring may introduce zero divisors). Let then $\mathfrak{D}$ be any commutative ring with unit element, and let again $\mathfrak{u}$ be a prime ideal in $\mathfrak{o}$. We may define the factor ring $\mathfrak{o} / \mathfrak{u}$ exactly in the same way as above, but we cannot so easily generalize the notion of the ring of quotients $\mathfrak{D}_{\mathfrak{u}}$. If there exist zero divisors outside $\mathfrak{u}$, these zero divisors cannot be used as denominators of fractions, which shows that the definition of $\mathfrak{p}_{\mathfrak{u}}$ cannot be extended verbatim. If we

Received by the editors September 4, 1943. 
consider only fractions whose denominators are not zero divisors and do not belong to $\mathfrak{u}$, we obtain a ring $\mathfrak{D}^{\prime}$; but $\mathfrak{D}^{\prime}$ fails in general to have the essential property of a ring of quotients, namely, of being a local ring in the sense of Krull (that is, the non-units in $D^{\prime}$ will not form an ideal). The object of this note is to construct a ring for which the essential properties of rings of quotients are preserved.

Throughout this paper we shall denote by o a Noetherian ring (that is, a ring in which the maximal condition for ideals is satisfied) with a unit element. Generalizing the problem of defining the ring of quotients of a prime ideal, we take any multiplicatively closed $^{1}$ subset $S$ of $\mathrm{D}$ which does not contain 0 (a set is said to be multiplicatively closed if the product of any two elements of the set belongs to the set; if we are concerned with a prime ideal $\mathfrak{t}$ in $\mathfrak{D}$, we take $S$ to be the complement of $\mathfrak{u}$ in $\mathfrak{D}$ ). There exists at least one primary ideal which does not meet $S$ (otherwise, 0 would belong to $S$ as we see at once by representing the zero ideal as an intersection of primary ideals). We shall denote by the intersection of all primary ideals in $\mathrm{D}$ which do not meet $S$.

Proposition 1. Let $\{0\}=q_{1} \cap \cdots \cap q_{h}$ be an irredundant representation of the zero ideal in $\mathrm{D}$ as an intersection of primary ideals, and let $\mathfrak{p}_{i}(1 \leqq i \leqq h)$ be the associated prime ideal of $\mathfrak{q}_{i}$. Assume that $\mathfrak{p}_{i} \cap S=\varnothing$ for $i \leqq g$, but not for $i>g$. Then $\xi=q_{1} \cap \cdots \cap q_{g}$.

It is clear that $\mathfrak{B} \subset \mathfrak{q}^{\prime}=\mathfrak{q}_{1} \cap \cdots n \mathfrak{q}_{g}$. Let $\mathfrak{v}$ be any primary ideal which does not meet $S$; we shall prove that $q^{\prime} \subset \mathfrak{v}$. Let $q^{\prime \prime}$ be the ideal $\mathfrak{q}_{a+1} \cap \cdots \cap \mathfrak{q}_{h}$. We have $\{0\}=\mathfrak{q}^{\prime} \cap \mathfrak{q}^{\prime \prime}=\mathfrak{q}^{\prime} \mathfrak{q}^{\prime \prime} \subset \mathfrak{v}$, whence $\mathfrak{q}^{\prime} \subset \mathfrak{v}: \mathfrak{q}^{\prime \prime}$. Let $\mathfrak{u}$ be the associated prime ideal of $\mathfrak{v}$; since $\mathfrak{v}$ contains some power of $\mathfrak{u}$, it follows from the multiplicatively closed character of $S$ that $\mathfrak{u}$ does not meet $S$. If $i>g$, the ideal $\mathfrak{p}_{i}$ meets $S$ and is therefore not contained in $\mathfrak{u}$. It follows ${ }^{2}$ that $\mathfrak{v}: \mathfrak{q}^{\prime \prime}=\mathfrak{v}$, whence $\mathfrak{q}^{\prime} \subset \mathfrak{v}$. Proposition 1 is thereby proved.

LEMma 1. Let $\mathfrak{p}$ be a prime ideal in $\mathrm{o}$, and let $\mathfrak{a}$ be an ideal contained in $\mathfrak{p}$. If $\mathfrak{q}$ is an ideal containing $\mathfrak{a}$, the statements " $\mathfrak{q}$ is primary for $\mathfrak{p}$ " and “ $\mathfrak{q} / \mathfrak{a}$ is primary for $\mathfrak{p} / \mathfrak{a}$ " are equivalent.

\footnotetext{
${ }^{1}$ It was $\mathrm{H}$. Grell who observed for the first time that, $S$ being any multiplicatively closed set of nonzero divisors in a ring, it is possible to associate with $S$ a ring of quotients, whose elements are the fractions whose denominators belong to $S$ (Cf. H. Grell, Beziehungen zwischen Ideale verschiedener Ringe, Math. Ann. vol. 97 (1926) p. 510). For the properties of these rings of quotients, cf. Krull, Idealtheorie (Ergebnisse der Mathematik) or my paper On the theory of local rings, Ann. of Math. vol. 44 (1943) p. 690.
}

2 Cf. van der Waerden, Moderne Algebra, vol. 2, chap. 12, p. 36. 
Lemma 1 follows trivially from the definitions.

The zero ideal in $\mathrm{o} / \mathbb{B}$ is the intersection of the primary ideals $\mathfrak{q}_{1} / \mathfrak{z}, \cdots, \mathfrak{q}_{g} / \mathfrak{z}$ whose associated prime ideals are $\mathfrak{p}_{1} / \mathfrak{z}, \cdots, \mathfrak{p}_{g} / \mathfrak{z}$. Let $S^{*}$ be the set of the residue classes modulo $\&$ of the elements of $S$; then $S^{*} \cap\left(\mathfrak{p}_{i} / \mathfrak{z}\right)=\varnothing(1 \leqq i \leqq g)$, which means that no element of $S^{*}$ is a zero divisor in $\mathfrak{D} / 8 .{ }^{3}$ We may therefore construct the ring of quotients $(\mathrm{o} / \mathrm{B})_{S^{*}}$ of $S^{*}$ with respect to the ring $\mathrm{o} / \mathrm{B}$.

DeFINITION 1. The ring $(\mathrm{o} / \mathrm{B})_{S^{*}}$ will be called the ring of quotients of $S$ with respect to $\mathrm{D}$. This ring will be denoted by $\mathrm{D}_{S}$.

This definition coincides with the usual one in the case where $S$ does not contain any zero divisor. We shall now prove that the essential properties of rings of quotients in the usual sense still hold in our case.

If $\mathfrak{a}$ is an ideal in $\mathfrak{D},(\mathfrak{a}+\mathfrak{B} / \mathfrak{b}) \mathfrak{o}_{S}$ is an ideal in $\mathfrak{D}_{S}$ which we shall denote symbolically by $a 0_{S}$ (in spite of the fact that $\mathfrak{D}$ is not in general a subring of $\boldsymbol{D}_{S}$, so that we cannot multiply elements of $\boldsymbol{D}$ by elements of $\left.\mathfrak{b}_{S}\right)$. If $\mathfrak{b}$ is any ideal in $\mathfrak{b}_{S}$, the set $\mathfrak{b} \cap(\mathfrak{o} / \mathfrak{b})$ may be written in the form $\mathfrak{a} / \mathfrak{b}$, where $\mathfrak{a}$ is an ideal in $\mathfrak{D}$ which contains $\mathfrak{B}$. We shall denote $\mathfrak{a}$ symbolically by $\mathfrak{b}_{\cap} \mathfrak{O}$ (although $\mathfrak{b}_{\cap} \mathfrak{D}$ is not a set theoretic intersection).

Proposition 2. If $\mathfrak{b}$ is any ideal in $\mathfrak{o}_{S}$, we have $\mathfrak{b}=\left(\mathfrak{b}_{n} \mathfrak{D}\right) \mathrm{o}_{\mathrm{S}}$.

Since $\mathfrak{D}_{S}$ is a ring of quotients of $\mathfrak{D} / \mathfrak{b}$, we have $\mathfrak{b}=(\mathfrak{b} \cap(\mathfrak{D} / \mathfrak{b})) \mathfrak{D}_{S} \cdot{ }^{1}$ Proposition 2 follows immediately from this formula.

Proposition 3. Let $\mathfrak{p}$ be a prime ideal in $\mathfrak{0}$, and let $\mathfrak{q}$ be primary for $\mathfrak{p}$. If $\mathfrak{q}$ meets $S$, we have $\mathfrak{p o}_{S}=\mathfrak{q D}_{S}=\mathfrak{D}_{S}$. If $\mathfrak{p}$ does not meet $S$, $\mathfrak{p o}_{S}$ is prime, $\mathfrak{q} \mathfrak{o}_{S}$ is primary for $\mathfrak{p o}_{S}$ and $\mathfrak{p o}_{S} \cap \mathbb{D}=\mathfrak{p}, \mathfrak{q} \mathfrak{o}_{S} \cap \mathbb{D}=\mathfrak{q}$.

If $q$ meets $S, q+\mathbb{z} / \mathbb{z}$ meets $S^{*}$, whence $\mathfrak{p o}_{S}=q \mathfrak{o}_{S}=\mathfrak{o}_{S}$. If $\mathfrak{p}$ does not meet $S$, the same holds for $\mathfrak{q}$, whence $s \subset q \subset p$. By Lemma $1, \mathfrak{p} / \mathfrak{z}$ is prime and $\mathfrak{q} / \mathbb{8}$ is primary for $\mathfrak{p} / \mathfrak{\&}$. Furthermore, $\mathfrak{p} / \mathfrak{\&}$ does not meet $S^{*}$. Proposition 3 follows therefore from the corresponding proposition which is known to hold for ordinary rings of quotients. ${ }^{1}$ We see also that, if $\mathfrak{p}$ does not meet $\mathbb{z}$, the formula $\mathfrak{q} \leftrightarrow q \mathfrak{q}_{S}$ establishes a one-to-one inclusion preserving correspondence between the primary jdeals for $p$ in $\mathfrak{D}$ and the primary ideals for $\mathfrak{p o}_{S}$ in $\mathfrak{o}_{S}$.

Proposition 4. Let $\mathfrak{a}=\mathfrak{b}_{1} \cap \cdots \cap \mathfrak{b}_{r}$ be an irredundant representation of an ideal $\mathfrak{a}$ in $\mathfrak{D}$ as an intersection of primary ideals. Let $\mathfrak{u}_{i}$ be the associated prime ideal of $\mathfrak{v}_{i}$, and assume that $\mathfrak{u}_{i}$ meets $S$ for $i>s$ but not for $i \leqq s$. Then we have $\mathfrak{a b}_{S}=\mathfrak{b}_{1} \mathfrak{D}_{S} \cap \cdots \cap \mathfrak{v}_{s} \mathfrak{D}_{S}$, and this is an irredundant representation of $\mathfrak{a 0}_{S}$ as intersection of primary ideals in $\mathfrak{o}_{S}$.

\footnotetext{
${ }^{3}$ If $s^{*} \in S^{*}$, we have $\{0\}: s^{*}(\mathfrak{D} / \mathbb{8})=\{0\}$ by the result quoted in footnote 2 .
} 
It is obvious that $\mathfrak{a}_{S} \subset \mathfrak{b}_{1} \mathfrak{D}_{S} \cap \cdots \cap \mathfrak{b}_{8} \mathfrak{D}_{S}$. Let conversely $a$ be any element of $\mathfrak{b}_{1} \mathfrak{D}_{S} \cap \cdots \mathfrak{b}_{s} \mathfrak{D}_{S}$. We know ${ }^{8}$ that $\mathfrak{b}_{1} \mathfrak{o}_{S} \cap \cdots \cap \mathfrak{b}_{s} \mathfrak{D}_{S}$ is equal to $\left(\mathfrak{b}_{1} / 8 \cap \cdots \cap \mathfrak{B}_{s} / \mathbb{8}\right) \mathfrak{o}_{S}$; it follows that $a$ may be written in the form $b^{*} / c^{*}$, where $b^{*} \in \mathfrak{b}_{1} / \mathbb{z} \cap \cdots \cap \mathfrak{b}_{s} / \mathfrak{z}$ and $c^{*} \in S^{*}$. If $i>s$, the ideal $\mathfrak{u}_{i}$ has an element $u_{i}$ in common with $S$; if $m$ is large enough, we have $u=\left(\prod_{i=s+1}^{r} u_{i}\right)^{m} \in \mathfrak{v}_{s+1} \cap \cdots \cap \mathfrak{v}_{r}$. Since $u_{i} \in S(s+1 \leqq i \leqq r)$, we have $u \in S$, whence $u^{*} \in S^{*}$, if $u^{*}$ is the residue class of $u$ modulo 8 . We may write $a=\left(u^{*} b^{*}\right) /\left(u^{*} c^{*}\right), u^{*} c^{*} \in S^{*}$. Let $b$ be any element of the residue class $b^{*}$ modulo $8 ;$ since $b^{*} \in \mathfrak{b}_{i} / \mathfrak{z}(1 \leqq i \leqq s)$, we have $b \in \mathfrak{b}_{1} \cap \cdots \cap \mathfrak{b}_{s}$, whence $u b \in \mathfrak{a}$ and $u^{*} b^{*} \in \mathfrak{a}+\mathbb{8} / \mathfrak{B}, a \in \mathfrak{a} \mathfrak{o}_{S}$. It is clear that none of the ideals $\mathfrak{b}_{i} / \&$ contains the intersection of the others; making use of a known result ${ }^{1}$ for ordinary rings of quotients, it follows that the representation $\mathfrak{a} \mathfrak{o}_{S}=\mathfrak{b}_{1} \mathfrak{D}_{S} \cap \cdots \mathfrak{b}_{8} \mathfrak{0}_{S}$ is irredundant.

We shall now consider more specifically the case where $S$ is the complement of a prime ideal $\mathfrak{u}$. The ring $\mathfrak{D}_{S}$ will then also be denoted by $\mathfrak{o}_{\mathfrak{u}}$. In that case, the ideal coincides with the intersection of all primary ideals for $\mathfrak{u}$. In fact, the set $S^{*}$ is clearly the complement of $\mathfrak{u} / \mathbb{B}$ with respect to $\mathfrak{D} / \mathbb{B}$; the ring $\mathfrak{D}_{S}$ is the ring of quotients (in the ordinary sense) of the prime ideal $\mathfrak{u} / \mathbb{\&}$ with respect to $\mathrm{D} / \mathbb{\&}$. It follows that $\mathfrak{u} \mathfrak{v}_{S}$ is the ideal of non-units in $\mathfrak{D}_{S}$, whence $\cap_{n-1}^{\infty}(\mathfrak{u} / \mathbb{Z})^{n} \mathfrak{D}_{S}=\{0\} .4^{4}$ For every $n$, the ideal $(\mathfrak{u} / \mathfrak{B})^{n} \mathfrak{D}_{S} \cap \mathbb{D} / \mathfrak{z}$ is a primary ideal for $\mathfrak{u} / \mathfrak{z}$ in $\mathfrak{D} / \mathfrak{z}$; it follows that the intersection of all primary ideals for $\mathfrak{u} / \mathfrak{b}$ is the zero ideal in $\mathbf{D} / \mathbf{8}$. Our assertion then follows from Lemma 1.5 At the same time, we see that $\mathfrak{D}_{\mathfrak{u}}$ is a local ring in the sense of Krull.

Assume now that $\mathrm{D}$ is a semi-local ring ${ }^{\boldsymbol{b}}$ and that $\mathfrak{u}$ is one of the maximal prime ideals in $\mathfrak{D}$. Let $\overline{\mathfrak{D}}$ be the completion of $\mathfrak{D}$; there corresponds to $\mathfrak{u}$ an idempotent $\epsilon$ in $\overline{\mathfrak{b}}$. We shall prove the following results:

PROPOSITION 5. The ring $\mathrm{D} / \mathbb{B}$ is isomorphic with the subring $\mathrm{D} \in$ of the ring $\overline{\mathbf{D}} \epsilon$. This isomorphism may be extended to an isomorphism of the completion of $\mathfrak{o}_{\mathfrak{u}}$ with $\overline{\mathbf{D}} \epsilon$.

The first statement will be proved if we show that 8 coincides with the set of elements $x \in \mathfrak{0}$ which satisfy the condition $x \epsilon=0$. If $x$ is any

${ }^{4}$ Cf. Krull, Dimensionstheorie in Stellenringen, J. Reine Angew. Math. vol. 179 (1938) p. 204 or my paper quoted in footnote 1.

5 This result, together with Proposition 1 above, yields a proof of a theorem of Krull; cf. Krull, Primidealketten in allgemeinen Ringbereiche, Sitzungsberichte der Heidelberger Akademie, 1928, p. 7.

${ }^{6} \mathrm{~A}$ semi-local ring is a Noetherian ring (that is, the maximal chain condition holds in the ring) in which there exist only a finite number of maximal prime ideals. For the proofs of the results on semi-local rings which are used in this paper, cf. my paper quoted in footnote 1. 
element of $\mathfrak{D}$, we may write $x=x \epsilon+x(1-\epsilon)$, and we know that $1-\epsilon \in \mathfrak{u}^{n} \overline{\mathfrak{D}}$ for every $n$. If $x \epsilon=0$, we have $x \in \mathfrak{U}^{n} \overline{\mathfrak{D}} \cap \mathfrak{D}=\mathfrak{u}^{n}$ for every $n$; in particular, $x$ belongs to every primary ideal for $\mathfrak{t}$, whence $x \in \mathbb{8}$. If $x \in \mathfrak{B}$, we have $x \in \mathfrak{u}^{n}$ for every $n$ ( $\mathfrak{u}^{n}$ is primary because $\mathfrak{u}$ is a maximal prime ideal), whence $x \epsilon \in \mathfrak{u}^{n} \bar{D} \epsilon$. Since $\mathfrak{u} \overline{\overline{0}} \epsilon$ is the ideal of non-units in $\bar{D} \epsilon$, it follows that $x \epsilon=0$.

If we identify $\mathfrak{D} / \mathbb{B}$ with $\mathfrak{D} \epsilon$, every element of $S^{*}$ is a unit in $\bar{D} \epsilon$. In fact, if $y \in S$, we have $y=y \epsilon+y(1-\epsilon), y(1-\epsilon) \in \mathfrak{H} \overline{0}$. If we had $y \in \mathfrak{H} \overline{0}$, we would have $y \in \mathfrak{u} \overline{0} \cap \mathcal{D}=\mathfrak{u}$, which is not the case. It follows that $\bar{D} \epsilon$ contains the ring $\mathfrak{D}_{\mathfrak{u}}$. The ring $\bar{D} \epsilon$ is a complete ring with $\mathfrak{t} \overline{\bar{D}} \epsilon$ as unique maximal prime ideal; it is clear that $\mathfrak{D \epsilon}$ (and, a fortiori, $\mathfrak{o}_{\mathfrak{u}}$ ) is dense in $\bar{D} \epsilon$. In order to prove that $\bar{D} \epsilon$ is the completion of $\mathfrak{D}_{\mathfrak{u}}$, it is sufficient to prove that $\mathcal{D}_{\mathfrak{u}}$ is topologically a subspace of $\bar{D} \epsilon$. We show first that $\mathfrak{u}^{n} \overline{\mathfrak{D}} \epsilon \cap \mathbb{D} \epsilon=\mathfrak{u}^{n} \mathfrak{D} \epsilon$ for every $n$. Let $x \epsilon(x \in \mathfrak{D})$ be an element of $\mathfrak{u}^{n} \overline{\mathrm{D}} \epsilon \cap \mathcal{D} \epsilon$; we have $x=x \epsilon+x(1-\epsilon) \in \mathfrak{u}^{n} \overline{\mathfrak{D}}$, whence $x \in \mathfrak{u}^{n} \overline{\mathfrak{D}} \cap \mathfrak{D}=\mathfrak{u}^{n}, x \epsilon \in \mathfrak{u}^{n} \epsilon$. The ideal $\mathfrak{u}^{n} \bar{D} \cap \mathfrak{D}_{\mathfrak{u}}$ is equal to $\left(\left(\mathfrak{u}^{n} \overline{\mathfrak{D}} \cap \mathfrak{D}_{\mathfrak{u}} \mathfrak{D}\right) \cap \epsilon\right) \mathfrak{D}_{\mathfrak{u}}=\left(\mathfrak{u}^{n} \mathfrak{D} \epsilon\right) \mathfrak{D}_{\mathfrak{u}}=\mathfrak{u}^{n} \mathfrak{D}_{\mathfrak{u}} ;$ Proposition 5 is thereby completely proved.

Princeton University 